\title{
Pharmacogenomics, a novel section in the European Journal of Human Genetics
}

\author{
Henk-Jan Guchelaar ${ }^{1}$
}

Received: 1 June 2018 / Accepted: 5 June 2018 / Published online: 2 July 2018

(c) European Society of Human Genetics 2018

Now 15 years ago, in 2003, the International Human Genome Sequencing Consortium proudly announced that the Human Genome Project had been completed. This endeavor raised high expectations of clinical application of genetics in the near future. In this context, three major areas are often highlighted as being the first such applications: diagnosis and treatment of rare diseases, ancestry tests for population genetics, and pharmacogenomics. For diagnosis of heritable diseases, such as growth and mental disorders, sequencing techniques are currently applied in routine settings in academic medical centers. In addition, there is growing interest in the use of biogeographical ancestry tests provided via the internet for building family trees and learning about ones origin. Also, there have been great advances in the use of pharmacogenomics in the recent years.

Indeed, drug response, both with respect to efficacy and side effects shows significant interpatient variability. The concept of interindividual differences in drug response was proposed as early as 1909 by Garrod in his book The Inborn Errors of Metabolism [1]. Today, the concept of pharmacogenomics, namely that variation in drug response is (at least partly) related to genetic variation, is widely recognized. Studies have shown that response rates to major therapeutic classes of drugs range from 25-60 percent [2], and pharmacogenomic biomarkers may not only help to better understand variability in drug response but also to increase safety and efficacy of drug treatment by individualizing therapy.

In the recent years, clinical guidelines for interpretation of pharmacogenomics test results have become available both in Europe and the United States [3-5] and this may

Henk-Jan Guchelaar

h.j.guchelaar@lumc.nl

1 Dept. Clinical Pharmacy \& Toxicology, Leiden University Medical Center, Leiden, The Netherlands fuel the clinical implementation of pharmacogenomics in routine patient care. Indeed, for example pharmacogenomics testing of variants in the DPYD gene as to individualize the dose of fluoropyrimidines used in the treatment of a variety of cancers has been implemented in some medical centers [6]. In addition, large scale pharmacogenomics implementation programs have been initiated in the US and the European Union.

At least eleven implementation initiatives in the United States of America have been funded over the last 5 years [7, 8]. The PREDICT program at Vanderbilt University Medical Center aims to establish a framework and infrastructure for pre-emptive incorporation of pharmacogenomics information in the electronic health record [9]. The US Pharmacogenomics Research Network, has initiated the Translational Pharmacogenetics Program aimed at implementing pre-emptive pharmacogenomic testing in several clinical US sites in a real-world setting [10]. In addition, the 1200 Patients Project initiated by the University of Chicago is aimed at determining the feasibility and utility of incorporating pre-emptive pharmacogenomic testing of a panel of pharmacogenes and to examine the impact of providing pharmacogenomics test results on prescribing decisions and patient outcome [11]. The Displaying and Integrating Genetic Information Through the Electronic Health Record (EHR) Action Collaborative (DIGITiZe) focuses on integrating pharmacogenomic testing results of TPMT and HLA-B*5701 into the electronic health record for patients receiving prescriptions for azathioprine and abacavir [12]. The PG4KDS at St Jude Children's Research Hospital in Memphis incorporates all CPIC drug pairs into the electronic health record and clinician decision support system [13]. The Clinical Implementation of Personalized Medicine through Electronic Health Records and Genomics -Pharmacogenomics (CLIPMERGE) project at Mount Sinai Medical Center aims at enrolling patients who have previously opted-in to the institution's biobank and to implement pharmacogenomic testing within the hospital [14]. The Personalized Medicine Program at the University 
of Florida and Shands Hospital has implemented CYP2C19 genotyping for patients who have been prescribed clopidogrel, as a pilot study. This project aims to expand on the implementation to a variety of other practice settings [15]. At Mayo Clinic in Rochester, the RIGHT initiative is aimed at developing best practices for the implementation of genetic sequence data into clinical systems [16].

By March 2016, the first large-scale, international pharmacogenomics implementation project 'Ubiquitous Pharmacogenomics' (U-PGx) has been initiated in Europe. The European Commission recently awarded a 15 million Euro Horizon 2020 grant to fund the Ubiquitous Pharmacogenomics Program 'Making actionable pharmacogenomics data and effective treatment optimization accessible to every European citizen' (www.upgx.eu). The project encompasses a randomized controlled trial in 8100 patients and will investigate the impact of pre-emptive pharmacogenomic testing of a panel of 13 pharmacogenes on patient outcomes in seven European countries [8].

All these initiatives have three similar approaches in common, as follows: 1) they implemented pre-emptive pharmacogenomic testing using either a single drug-gene combination, a panel of pharmacogenes, or NGS in clinical care, 2) they embedded the pharmacogenomics results within the (electronic) health record preferably combined it with clinical decision support systems and 3) they used available clinical pharmacogenomics guidelines to guide drug selection and dosing.

Nevertheless, pharmacogenomics is still a novel and emerging discipline and will be further developed over the next years. The understanding of the genetic impact on drug response is still incomplete and warrants further research. For this reason, the European Journal of Human Genetics launched a section 'Pharmacogenomics'. With this initiative, we hope to share cutting edge research papers on pharmacogenomics with readers of our journal and provide a platform to researchers to publish their work in this dynamic field.

\section{References}

1. Garrod AE. The inborn errors of metabolism. London: Oxford University Press; 1909.
2. Spear BB, Heath-Chiozzi M, Huff J. Spear, Clinical application of pharmacogenetics. Trends Mol Med. 2001;7:201.

3. Swen JJ, Nijenhuis M, de Boer A, et al. Pharmacogenetics: from bench to byte--an update of guidelines. Clin Pharmacol Ther. 2011;89:662-73.

4. Swen JJ, Wilting I, de Goede AL, et al. Pharmacogenetics: from bench to byte. Clin Pharmacol Ther. 2008;83:781-7.

5. Caudle KE, Klein TE, Hoffman JM, et al. Incorporation of pharmacogenomics into routine clinical practice: the Clinical Pharmacogenetics Implementation Consortium (CPIC) guideline development process. Curr Drug Metab. 2014;15:209-17.

6. Lunenburg CA, van Staveren MC, Gelderblom H, Guchelaar HJ, Swen JJ. Evaluation of clinical implementation of prospective DPYD genotyping in 5-fluorouracil- or capecitabine-treated patients. Pharmacogenomics. 2016;17:721-9.

7. van der Wouden CH, Swen JJ, Samwald M, Mitropoulou C, Schwab M, Guchelaar HJ. A brighter future for the implementation of pharmacogenomic testing. Eur J Hum Genet. 2016;24:16 58-60.

8. van der Wouden $\mathrm{CH}$, Cambon-Thomsen $\mathrm{A}$, Cecchin $\mathrm{E}$, et al. Ubiquitous Pharmacogenomics Consortium. Implementing pharmacogenomics in Europe: design and implementation strategy of the ubiquitous pharmacogenomics consortium. Clin Pharmacol Ther. 2017;101:341-58.

9. Pulley JM, Denny JC, Peterson JF, et al. Operational implementation of prospective genotyping for personalized medicine: the design of the Vanderbilt PREDICT project. Clin Pharmacol Ther. 2012;92:87-95.

10. Shuldiner AR, Relling MV, Peterson JF, et al. The Pharmacogenomics Research Network Translational Pharmacogenetics Program: overcoming challenges of real-world implementation. Clin Pharmacol Ther. 2013;94:207-10.

11. O'Donnell PH, Bush A, Spitz J, et al. The 1200 patients project: creating a new medical model system for clinical implementation of pharmacogenomics. Clin Pharmacol Ther. 2012;92:446-9.

12. Bell GC, Crews KR, Wilkinson MR, et al. Development and use of active clinical decision support for preemptive pharmacogenomics. J Am Med Inform Assoc. 2014;21:e93-e99.

13. Fernandez CA, Smith C, Yang W, et al. Concordance of DMET plus genotyping results with those of orthogonal genotyping methods. Clin Pharmacol Ther. 2012;92:360-5.

14. Gottesman O, Scott SA, Ellis SB, et al. The CLIPMERGE PGx Program: clinical implementation of personalized medicine through electronic health records and genomicspharmacogenomics. Clin Pharmacol Ther. 2013;94:214-7.

15. Johnson JA, Burkley BM, Langaee TY, Clare-Salzler MJ, Klein TE, Altman RB. Implementing personalized medicine: development of a cost-effective customized pharmacogenetics genotyping array. Clin Pharmacol Ther. 2012;92:437-9.

16. Bielinski SJ, Olson JE, Pathak J, et al. Pre-emptive genotyping for personalized medicine: design at the right drug, right dose, right time with genomic data for individualized treatment protocol. Mayo Clin Proc. 2014;89:25-33. 